Sección monográfica / Monographic section

\title{
Innovación y distinción bajo el objetivo de la calidad en la producción de frutos rojos en Huelva
}

\author{
Innovation and distinction under the perspective of quality in \\ Huelva's berries production sector
}

\author{
Alicia Reigada* \\ Universidad de Sevilla, España. \\ aliciareigada@us.es \\ Juana Moreno Nieto \\ Universidad de Cádiz, España. \\ juana.moreno@uca.es

\section{Carmen Mozo \\ Universidad de Sevilla, España. \\ cmozo@us.es}

Recibido / Received: 06/05/2020 Aceptado / Accepted: 28/09/2020

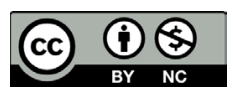

\section{RESUMEN}

En un contexto marcado por el aumento de la competitividad a nivel internacional y el poder de la gran distribución, el sistema de producción de frutos rojos de Huelva ha tenido que llevar a cabo diferentes ajustes con el fin de mantenerse en las cadenas agrícolas globales. Este artículo examina cómo se moviliza y configura un concepto de calidad, asociado a las ideas de innovación y distinción, en el marco de dos estrategias desarrolladas por el sector productivo en el último periodo: la especialización en productos únicos y saludables y la investigación varietal impulsada desde el ámbito local. El análisis se apoya en una metodología cualitativa basada en la técnica de la entrevista en profundidad.

Palabras clave: calidad; cadenas globales agrícolas; frutos rojos; producción; estrategias.

\section{ABSTRACT}

In a context characterized by an increase of international competitiveness and the concentration of power by big retailers, Huelva's berries sector has implemented several measures in order to remain in the global agri-food supply chains. This paper analyses how the concept of quality, which is linked to the notion of innovation and distinction, is defined and shaped within the framework of two strategies recently developed within the sector. These are specialization in unique and healthy products and locally driven variety research. The analysis is supported by a qualitative methodology based on in-depth interviews.

Keywords: quality; global agri-food supply chains; berries; production; strategies

\footnotetext{
*Autor para correspondencia/ Corresponding author: Alicia Reigada, aliciareigada@us.es
}

Sugerencia de cita / Suggested citation: Reigada, A., Moreno, J. y Mozo, C. (2021). Innovación y distinción bajo el objetivo de la calidad en la producción de frutos rojos en Huelva. Revista Española de Sociología, 30 (1), a17. https://doi.org/10.22325/fes/res.2021.17 


\section{INTRODUCCIÓN}

El cultivo industrial de fresas y otros frutos rojos en Huelva (Andalucía), que se extiende a lo largo de 12.000 ha en el litoral de la provincia, constituye uno de los principales enclaves agroexportadores de Europa. Cimentado en sus orígenes sobre un modelo dominado por la pequeña propiedad de carácter familiar, se caracteriza por la intensificación y especialización de la producción fuera de temporada, el empleo de insumos industriales, la innovación tecnológica, la orientación exportadora, el uso intensivo de capital y recursos naturales, la salarización del trabajo y la movilidad laboral. La última década ha venido acompañada de importantes cambios en la organización de la producción, una parte fundamental de estas transformaciones está relacionada con la cuestión de la calidad. En un contexto marcado por el aumento de la competitividad a nivel internacional y el poder de la gran distribución (Burch y Lawrence, 2009; Lawrence y Dixon, 2016), el sistema de producción de fresas de Huelva ha tenido que llevar a cabo diferentes ajustes con el fin de integrarse y mantenerse en las cadenas agrícolas globales.

Como en otros territorios rurales especializados en la producción en fresco, la calidad ha pasado a ocupar un lugar estratégico en esta agricultura. "Los frutos rojos de Huelva ya no son solo volumen, sino calidad" (Lozano, 2019), afirma Raúl Martínez, gerente de la multinacional Driscoll's de España Operaciones, afincada en la zona fresera de Moguer (Huelva). "Encarar una etapa más selectiva", que permita potenciar este valor añadido haciendo de la calidad una ventaja competitiva, es la apuesta de esta multinacional del sector agroalimentario, y del conjunto de cooperativas y empresas familiares dedicadas a la producción de frutos rojos. Inversión de capital, investigación en biogenética, desarrollo de la telemática, adaptación a las certificaciones estandarizadas, ampliación de la oferta de variedades y formatos, profesionalización, desarrollo de técnicas de mercadotecnia, reorganización de los procesos de trabajo y de los mecanismos de control son algunos de los ajustes que exige esta nueva fase.

El peso que progresivamente ha ido adquiriendo la calidad en la gobernanza de las cadenas agroalimentarias se corresponde con la proliferación de investigaciones que ahondan en las características, dispositivos y transformaciones que conlleva. Numerosos estudios subrayan cómo la calidad se ha convertido en un mecanismo que aumenta el control de la gran distribución sobre el sistema productivo y los procesos de trabajo a través, especialmente, de normas privadas de regulación y certificación implementadas en el ámbito de la seguridad alimentaria, el medio ambiente y el trabajo (Konefal, Mascarenhas y Hatanaka, 2005; Bonanno y Cavalcanti, 2012; Ransom, Bain y Higgins, 2013; Moraes y Cutillas, 2014). Al igual que en otros sectores y regiones, en la agricultura onubense se han implantado las principales certificaciones privadas ${ }^{1}$ que regulan el sector agroalimentario a nivel internacional: Global GAP, IFS (International Featured Standards) y BRC (British Retail Consortium).

Aunque en la actualidad las certificaciones estandarizadas privadas constituyen un dispositivo decisivo en la gestión y definición de la calidad, el conjunto de procesos y actores que interviene es más amplio. Los estudios preocupados por analizar cómo afectan al sistema alimentario los cambios derivados de la globalización y el postfordismo han prestado atención a otras dimensiones que integran el campo de la calidad. En el marco de los análisis sobre la dualización de la producción y el consumo, la especialización territorial, la flexibilidad productiva y laboral, la segmentación de los mercados y los procesos de diferenciación, estas investigaciones han mostrado cómo la calidad deviene un parámetro decisivo de competitividad en la llamada agricultura postfordista o flexible

1 Además de la certificación pública Producción Integrada de Andalucía (PIA), que se introdujo a inicios de los años dos mil. 
(Friedland, 1994; Lara, 1998; Pedreño, 1999). Como sostiene Marsden (1997, p. 170), “los mercados agroalimentarios están siendo re-regulados en torno a las demandas de los consumidores del norte y los supermercados", y el valor de los alimentos está siendo fijado a partir de aspectos relacionados con la calidad (basados en desarrollos tecnológicos y redes de conocimiento).

Entendida, como plantea la teoría de la convención, como un constructo social (Renard, 2003), la noción de calidad no se configura en abstracto ni los criterios que la definen son universales, sino que son las relaciones sociales, las prácticas y las creencias de los diferentes actores las que dan lugar a las convenciones sobre calidad (Marsden y Arce, 1995; Ponte y Gibbon, 2005; Martínez, 2019). Como recuerda Narotzky (2004), las mercancías no pueden ser abstraídas de las relaciones sociales que las producen y de los significados que adquieren en el marco de esas relaciones. Ello explica que la noción de calidad pueda implicar percepciones y características incluso opuestas en función de los productos o los nichos de mercado a los que vaya dirigido (Lara, 1999). Así, por ejemplo, el respeto al medio ambiente o la ausencia de pesticidas en los productos biológicos constituyen una marca de calidad para determinadas capas de la población, al tiempo que la presentación en envases de plástico o las frutas de formas estandarizadas y homogéneas, propias de la agricultura intensiva, también son consideradas rasgos de calidad (Moreno, 2016). Competir en calidad en el sector de la producción intensiva en fresco significa asignar a estos alimentos atributos distintivos, y a veces contradictorios, entre los que sobresalen: las propiedades naturales, nutricionales y saludables; la forma, el calibre, el color, el sabor, la textura y la conservación; la diversificación de envases y marcas; y la incorporación de productos nuevos como los frutos tropicales y exóticos (Farruggia, Crescimannoa, Galatia y Tinervia, 2016; Friedland, 1994; Pedreño, 2001).

Este artículo analiza cómo estos atributos de calidad se convierten en un factor clave de competitividad en las estrategias empresariales actuales del sistema de producción de frutos rojos de Huelva. En particular, examina la apuesta reciente del sector por la especialización en productos únicos y saludables y por la investigación varietal desde lo local, y cómo en el marco de estas estrategias se construye y moviliza un concepto de calidad asociado a las ideas de innovación y distinción propias del postfordismo.

El artículo está organizado en cuatro apartados. El primero explica la metodología y el trabajo de campo en que se apoya el análisis. A continuación, se presentan algunas claves teórico-contextuales que permiten situar las estrategias de diferenciación del sector partiendo de un enfoque que articula la dimensión global y territorial. El tercer apartado examina cómo se incorpora y el significado que adquiere el principio de calidad en dos estrategias empresariales del sistema de frutos rojos de Huelva: la especialización en productos definidos por su unicidad, novedad y propiedad nutricional y los ensayos en centros de I + D locales de variedades consideradas de alta calidad. El artículo termina con un apartado que recoge las conclusiones finales.

\section{METODOLOGÍA}

La metodología utilizada en esta investigación es de carácter cualitativo y ha consistido principalmente en la realización de entrevistas en profundidad a diferentes actores del sector onubense de frutos rojos, así como a responsables de empresas consultoras y auditoras externas que participan de la gestión de los sistemas de calidad en el sector ${ }^{2}$. De manera complementaria, se han analizado los contenidos de las páginas web de las

2 Los nombres citados en el artículo son ficticios, tanto en el caso de las personas entrevistadas como de las cooperativas y empresas. En el caso de las entrevistas publicadas en revistas vinculadas al sector y en prensa, o de otro tipo de información pública, se han respetado los nombres reales. 
empresas en las que hemos realizado entrevistas y de artículos publicados en revistas especializadas y portales web vinculados al sector ${ }^{3}$. El trabajo de campo fue realizado entre abril de 2019 y abril de 2020. Asimismo, el artículo se apoya en el bagaje y los resultados de dos proyectos de investigación previos realizados por las diferentes autoras en el cultivo de la fresa en Huelva entre 2006 y 2016.

Se trata de una investigación en curso y, en esta primera fase, se han realizado 15 entrevistas, en su mayoría a personal técnico de las áreas de calidad, recursos humanos, seguridad alimentaria y marketing de las cooperativas y empresas comercializadoras del sector y, en menor medida, a productores agrícolas. Asimismo, se han realizado dos entrevistas a responsables de empresas consultoras y auditoras externas que trabajan de manera estrecha con agentes del sector. Las entrevistas han tenido, a excepción de tres, un carácter presencial, por lo que algunas se han visto complementadas con visitas a las explotaciones agrícolas y almacenes de acondicionamiento.

La opción por privilegiar en esta fase las entrevistas de perfil técnico de la rama de la comercialización y acondicionamiento de fruta está relacionada con que es en este ámbito donde se desarrollan y promueven las distintas estrategias que se han puesto en práctica en estos últimos años en el sector para construir la calidad de la producción de frutos rojos en Huelva. De hecho, se trata de un segmento clave de la cadena puesto que actúa de transmisor y supervisor en la implementación de estas estrategias en finca. Por esto, a pesar del número limitado, las entrevistas realizadas han permitido identificar las principales estrategias y propuestas existentes en torno a la calidad hoy día en el sector, así como los significados y lógicas que acompañan a estos procesos. Ello se ha visto complementado con el análisis de las páginas web y las revistas vinculadas al sector, que ha posibilitado ahondar en los discursos que movilizan patronal y empresas en torno a la cuestión de la calidad y desgranar algunas de las actuaciones que se están desarrollando recientemente en la materia.

\section{GLOBALIZACIÓN, TERRITORIALIZACIÓN Y DIFERENCIACIÓN: CLAVES TEÓRICO- CONTEXTUALES}

El enfoque de la nueva economía política de la agricultura (McMichael, 1996; Friedland, 2004; Marsden, 2012) ha situado la dimensión territorial como una variable explicativa de las relaciones de producción y consumo que configuran la acumulación de capital en los distintos regímenes alimentarios (Friedmann y McMichael, 1989). Esta dimensión está muy presente en los estudios sobre innovación, diferenciación y acumulación flexible en el postfordismo, preocupados por abordar estos procesos articulando las dinámicas de globalización y territorialización (Harvey, 1998; Pedreño, 2001; Veltz, 1999).

El análisis territorial también cuenta con una larga trayectoria en los estudios sobre las agriculturas mediterráneas en nuestro país (Delgado, 2002; Lacomba, 1997; Pedreño, 1999). Desde una perspectiva atenta a la historia local de los cultivos, estas investigaciones han mostrado la concreción en los contextos locales de dinámicas globales como la transformación de las técnicas y tipos de cultivo, la industrialización de la agricultura, la flexibilidad productiva o la fragmentación laboral. Aplicar este enfoque al análisis de los cambios recientes en las estrategias de especialización productiva e investigación varietal en el cultivo de la fresa en Huelva permitirá situar algunas claves contextuales fundamentales.

3 Entre las que destacan la revista Mercados, la revista Origen y el portal de noticias FreshPlaza.es. 
La especialización del territorio en la producción de alimentos frescos fuera de temporada constituye la base de la agricultura industrial en Huelva desde su implantación en los años sesenta del siglo XX. Se trata de un fenómeno global enmarcado en la división territorial e internacional del trabajo y en los consecuentes procesos de especialización regional y concentración del capital (McMichael, 2011). En el caso que nos ocupa, esta especialización se ha concretado en la expansión del monocultivo de fresas, en base a un modelo apoyado, en las primeras etapas, en la pequeña propiedad de carácter familiar, y caracterizado en los últimos veinte años por el aumento del tamaño y la concentración de la propiedad. Convertido en uno de los grandes líderes de exportaciones de fresas a nivel internacional ${ }^{4}$, debe retenerse que bajo este modelo el territorio andaluz asume el papel de extractor de recursos naturales y suministrador de materias primas y ciertos bienes agroalimentarios (Delgado, 2002). Esto es, se inserta en una estructura, la de la cadena de valor, fuertemente jerarquizada, en la que el sector se sitúa en una posición dependiente respecto de las empresas extranjeras que dominan la investigación y el desarrollo tecnológico, las industrias y multinacionales que suministran los insumos y paquetes tecnológicos, y las cadenas de distribución que controlan los precios y los canales de comercialización.

Durante décadas la estrategia productiva basada en el monocultivo generó holgados beneficios a las empresas, en un periodo en el que aumenta la clientela que consume productos en fresco y el valor añadido de la producción orientada a nichos de mercado diferenciados (Friedland, 1994), y en el que la relación entre costes de producción e ingresos era favorable al sector. La entrada en el siglo XXI viene marcada, sin embargo, por una acentuación del desequilibrio que empieza a experimentar esta relación a finales de los noventa, como resultado del incremento progresivo de los costes de producción y el descenso del precio al que los productores venden la fruta (Delgado y Aragón, 2006)5.

Este desequilibrio en la balanza converge con otros factores que inciden a nivel global, como el incremento de la competitividad e incertidumbre de los mercados agroalimentarios, el aumento del poder de la gran distribución, el ascenso de otros países productores de fresas en los circuitos de exportación (como Marruecos, Alemania o Italia) y la profundización de las tendencias de diferenciación propias del postfordismo, entre las que sobresale la preocupación por la calidad de los alimentos. Como respuesta a este escenario, y para ajustarse a las nuevas exigencias de los mercados ${ }^{6}$, en la última década el sector ha apostado por actualizar la especialización basada en el monocultivo de fresas en el marco de una estrategia más amplia de diversificación y especialización en productos únicos y saludables.

Al igual que en otros territorios agroexportadores, la estrategia de competitividad de las empresas de frutos rojos en Huelva va a intentar combinar la búsqueda de economías de escala y economías de variedad y diferenciación del producto (calibre, color, apariencia, empacado, marcas, nuevos productos) (Pedreño, 2001). Como sostiene el autor, esta última supone el pasó de la lógica del precio, propia de la producción fordista estandarizada, a la lógica de la calidad. Un cambio que ya apuntaba Roseberry (1996) en su estudio sobre las formas de distinción en la producción y consumo de café, en el que analiza el paso a la

4 Según Eurostat, en 2018 España produjo 344,68 miles de toneladas de fresa (el 27\% de la fresa producida en la UE-28), seguido de Polonia (16,2\%) y Alemania (11,2\%). Andalucía produce el $97 \%$ de la fresa producida en el territorio nacional (Junta de Andalucía, 2019).

5 La evolución del precio medio (por kilo) resulta ilustrativa al respecto: 1,2 € en 1993, 0,75 en 2003 (Bergeron y Darpeix, 2004 ) y 0,92 en 2013 (Junta de Andalucía, 2019). Además, la cadena de valor de la fresa de Huelva a principios del siglo XXI muestra que la gran distribución se apropia de un $73 \%$ del valor final pagado por el consumidor. De la parte restante recibida por el productor (un $27 \%$ ), el $20 \%$ se destina a pagar los inputs agroindustriales (Delgado y Aragón, 2006, p. 463).

6 Este artículo no tiene como objeto de estudio el análisis del trabajo, si bien debe subrayarse que la intensificación, precarización y segmentación del trabajo ha sido una estrategia fundamental en la adaptación y sostenimiento del modelo. Ver: Moreno (2009) y Reigada $(2012,2016)$. 
venta en función de una idea de calidad ligada a la imagen de unicidad del producto (desde el punto de vista del sabor, olor, apariencia). Esta búsqueda de economías de variedad y diferenciación del producto conlleva, además de la adaptación de la producción material de alimentos a las nuevas demandas y gustos distinguidos, el crecimiento de la dimensión simbólica de la economía (Bourdieu, 1979), que hace crecer exponencialmente el mundo de la imagen, los significados y las apariencias (Alonso, 2007).

La investigación varietal, orientada a aumentar la productividad de la planta y a mejorar la presencia y conservación de los frutos, es otro de los pilares que sostiene el modelo desde sus inicios. Al igual que la especialización productiva, este ámbito ilustra de manera ejemplar las relaciones de dependencia territorial. La investigación en biogenética, que tiene como propósito suplantar las variedades autóctonas de fresas por variedades preparadas en centros de experimentación y laboratorios, ha estado marcada por el dominio estadounidense (Salas y Flores, 1985), en particular de la Universidad de California y la Universidad de Florida. Tioga, Douglas y Chandler dominaron el mercado en los años ochenta, siendo relevadas por Oso Grande a principios de los noventa y, posteriormente, por Camarosa y Ventana, algunas de las variedades más plantadas en la última década.

A principios del siglo XXI entra con cierta fuerza la empresa de I + D + I Plantas de Navarra S.A (Planasa), hoy integrada en una compañía internacional con notable poder en el mercado global de variedades. Si la investigación varietal ha permitido al sector productivo de Huelva desarrollar una de las principales estrategias por las que ha apostado, la intensificación productiva, lo ha hecho a costa de ahondar en la posición subordinada que ocupa en la cadena de valor, tanto desde el punto de vista monetario, al quedar sujeto al pago de royalties, como de los conocimientos, al quedar supeditado al saber científicotécnico controlado, además, por empresas extranjeras y compañías transnacionales.

El impulso dado en las dos últimas décadas al ensayo de variedades de fresas desde el ámbito local responde al objetivo de superar esa dependencia tecnológica (Silva, 2011). La apuesta del sector productivo de Huelva por invertir en I + D + I para obtener variedades creadas y patentadas desde el ámbito local se desarrolla en un contexto en el que las plusvalías se concentran cada vez más en los eslabones que dominan la innovación tecnológica y la investigación aplicada, entre los que se hallan las compañías que generan nuevas semillas (Castells y Hall, 1994). En una coyuntura, también, en la que la investigación varietal pasa a ser considerada un factor importante de calidad. Como sostienen Sorj y Wilkinson (1994), la preocupación por la calidad constituye un elemento determinante de la innovación en biotecnología en la industria alimentaria. Observan que al tiempo que se demandan biotecnologías que garanticen la calidad de las materias primas (entendida como natural, saludable), limitando el uso de productos químicos y aumentando los controles de toxicidad, se introducen colores y sabores de origen químico que compensan la pérdida de los originarios como consecuencia del tratamiento industrial (Sork y Wilkinson, 1994). Una paradoja que también apunta Martínez $(2014,2019)$ cuando muestra cómo, en el conjunto de concepciones sobre la calidad en el sector de los lácteos, se destaca como relevante el aspecto natural de la producción de alimentos al tiempo que la tecnología y el conocimiento científico se consideran elementos determinantes para la obtención de esa calidad.

La biotecnología pasa a convertirse, así, en un instrumento decisivo en la construcción de la calidad, tanto de aquellas variables ligadas a lo natural, la salud y la seguridad alimentaria, como de aquellas otras asociadas al calibre, la forma, el color, el sabor, la textura y la conservación. De manera que, junto a la sofisticación de las técnicas de cultivo y la aplicación de las nuevas tecnologías de la información a la producción, las innovaciones varietales han permitido el desarrollo de una agricultura de precisión, capaz de adaptarse a los diferentes requerimientos de calidad de los distintos mercados (Goodman y Watts, 1997, p. 20). 
Situar en la historia del cultivo de frutos rojos de Huelva las claves para comprender los factores y procesos que conducen a una profundización de las estrategias de diferenciación y especialización del sector, en aras de responder a los requerimientos de los mercados, permite contextualizar las transformaciones que entraña la nueva fase centrada en la calidad. A continuación, se examina cómo se expresan y materializan algunas de estas transformaciones.

\section{LA CONSTRUCCIÓN DE LA CALIDAD EN EL MARCO DE LAS ESTRATEGIAS DEL SECTOR}

\section{La apuesta por productos únicos y saludables: la especialización del sector bajo el objetivo de la calidad}

A finales de los años cincuenta del pasado siglo el abogado sevillano Antonio Medina introdujo en la finca Las Madres, ubicada en Moguer, la tecnología agraria más avanzada procedente de California y de los campos israelitas, abriendo con ello el camino hacia el cultivo intensivo de fresas en Huelva. La apuesta por la innovación acompañará a esta empresa sevillana desde los orígenes del cultivo hasta la actualidad. Esto es, desde los primeros avances en las técnicas de cultivo bajo plástico en tierras arenosas y el transporte frigorífico, hasta la experimentación con cultivos novedosos en Andalucía que permitan ampliar los canales actuales de exportación. Aplicación de la industria a la agricultura, avance tecnológico y novedad son tres componentes de la idea de innovación que manejan, entendida ésta como el motor para la expansión comercial. Rocío Medina, hija de Antonio Medina y presidenta del grupo empresarial, coincide en situar la innovación y el desarrollo como la clave del éxito, y lo vincula, en la fase actual, a la diferenciación: "Buscamos hacer algo que no haga nadie para diferenciarnos" (Fernández, 2018). Es esta búsqueda la que llevó al grupo a reducir la producción de fresas e introducir nuevos tipos de cultivos ya a finales de los ochenta, el periodo de mayor expansión y rentabilidad económica del monocultivo de fresas. Convertido en el primer exportador de Europa de caqui sharoni, el Grupo Medina es representativo de los valores que orientan una de las principales estrategias productivas desde las que se construye la idea de calidad: la especialización de la producción a partir de la diferenciación.

La diversificación de los cultivos es una de las prácticas impulsadas en el marco de esta estrategia. En las últimas dos décadas el sector productivo ha optado por la diversificación como respuesta -entre otras posibles- al techo de rentabilidad del monocultivo de fresas. Esta diversificación se basa fundamentalmente en la introducción de otros frutos rojos cultivados de forma intensiva, como el arándano, la frambuesa y, en menor medida, la mora.

Tabla 1. Evolución de la superficie de frutos rojos en Huelva.

\begin{tabular}{|c|c|c|c|c|c|c|c|}
\hline CULTIVOS & $2010 / 11$ & $2011 / 12$ & $2012 / 13$ & $2013 / 14$ & $2014 / 15$ & $2015 / 16$ & $2016 / 17$ \\
\hline $\begin{array}{c}\text { Fresa } \\
\text { Arándano } \\
\text { Frambuesa } \\
\text { Mora } \\
\text { Total }\end{array}$ & $\begin{array}{c}6.400 \\
763 \\
955 \\
51 \\
8.169\end{array}$ & $\begin{array}{c}6.400 \\
777 \\
1.200 \\
50 \\
8.427\end{array}$ & $\begin{array}{c}6.830 \\
964 \\
1.140 \\
57 \\
8.991\end{array}$ & $\begin{array}{l}6.980 \\
1.150 \\
1.300 \\
70 \\
9.500\end{array}$ & $\begin{array}{c}6.304 \\
1.470 \\
1.560 \\
90 \\
9.460\end{array}$ & $\begin{array}{c}5.860 \\
1.953 \\
1.815 \\
130 \\
9.758\end{array}$ & $\begin{array}{c}5.400 \\
2.538 \\
1.932 \\
160 \\
10.030\end{array}$ \\
\hline
\end{tabular}

Fuente: Elaboración propia a partir de Freshuelva (2017, p. 34).

Consumidos en España hasta hace poco en momentos puntuales y especiales, estos frutos empiezan a extenderse en los mercados nacionales siguiendo los patrones de 
consumo postfordista de los países del centro y norte de Europa, donde se normalizaron hace un tiempo. Como ha revelado un estudio empírico reciente sobre las percepciones y comportamientos de los consumidores respecto a los frutos rojos, los factores de calidad atribuidos a estos frutos, en particular sus beneficios para la salud, su sabor y color, desempeñan un papel significativo en el creciente interés que han suscitado en los últimos años (Farruggia et al., 2016). Sus propiedades nutricionales y saludables, su presencia (tamaño, calibre, forma, brillo y color), su aroma y sabor, el carácter fresco y el cuidado que requiere su recolección, envasado, transporte y conservación, son las principales cualidades atribuidas a estos frutos y son potenciadas desde el sector a través de su imagen de marca.

Especialización y diferenciación son, para el grupo Extra Fruit, la base para dar un nuevo impulso a su expansión comercial a nivel internacional. Constituido en 2013 a partir de una estrategia de concentración empresarial, Extra Fruit es resultado de la unión de cinco empresas de carácter familiar con el objetivo de comercializar de forma conjunta y cubrir la especialización en una gama amplia de berries de alta calidad. La presentación que ésta y otras empresas hacen de los frutos que cultivan muestra de forma ilustrativa cómo la idea de calidad es movilizada en un marco comercial y cultural caracterizado por el uso intensivo de recursos comunicativos que apelan al valor de la diferencia y la unicidad. Estos valores son construidos, en buena medida, a partir de las cualidades antes apuntadas:

\begin{abstract}
La frambuesa, el sabor aterciopelado. Su precioso color rosado y textura aterciopelada hacen de ella una fruta única y de gran calidad (...). La mora es nuestra joya negra. Nuestro cultivo con un sistema único, nos garantiza una producción durante todo el año a través de variedades especiales, que nos proporcionan moras de gran calidad con aspecto globular y color púrpura oscuro, casi negro brillante, que posee un profundo sabor dulce con matices ácidos (web del grupo empresarial Extra Fruit).

Y defectos de calidad, pues lo típico, de daños superficiales, que el cáliz este verde, que no esté el cáliz seco. El cáliz es el... lo de arriba. Y a nivel de que no se marque mucho las pepitas, que... por ejemplo, cosas tan de eso, como que la punta de semilla, que no tenga acumulación de semilla en la fresa debajo del todo, sino que sea uniforme y que no tenga forma de pala, o que sea siempre cónica (Lucía Ruíz, técnica de calidad de Fresas Moreno).
\end{abstract}

En este objetivo por "aprender como sector a transmitir a los consumidores europeos las particularidades de nuestra fresa para que se vea como algo único", como subraya Carlos Cumbreras, gerente de la cooperativa Grufesa (Pitarch, 2015), se inscribe también la tendencia reciente a destacar las ventajas nutricionales y saludables de los frutos rojos. La apuesta por unos frutos que son pensados como "pequeñas píldoras de salud" constituye una de las estrategias comerciales por las que el sector trata de posicionarse en el mercado:

\footnotetext{
Continuamente surgen nuevos estudios que ponen sobre la mesa las extraordinarias propiedades de estas frutas, pequeñas píldoras de salud (Juan Báñez, Director General de Cuna de Platero, citado en Redacción, 2016).
}

En toda esta, toda esta historia de cómo conseguir posicionar el producto, el tema de la salud, nosotros estamos trabajando muy, de una forma muy intensa en eso ¿no? Con la, eh, tenemos un convenio con la Asociación Nacional de Cardiología, de bueno, para defender, para ver muchos congresos, muchas jornadas que se organizan para defender que, bueno, que es un producto, las berries, que van muy bien en ese sentido ¿no? De una buena salud, de una buena salud desde el punto de vista del corazón, en fin, eso sería un poco, es un poco el caballo de batalla que vengo de defender. (...) Y se vendería solo, crear poco a poco esa cultura (Antonio López, técnico de calidad de Onuberries).

Esta práctica se ha acentuado en los últimos cinco años, periodo en el que se han incorporado entradas específicas con la rúbrica "Salud" en las páginas web de las 
empresas y cooperativas y se suceden campañas y acciones promocionales que insisten en las ventajas saludables y nutricionales de los frutos rojos. De especial relevancia en estas iniciativas es el apoyo de la administración pública y el recurso creciente al aval de la ciencia, en concreto de especialistas médicos que dotan de autoridad los discursos del sector. Así lo refleja la participación de Antonio Escribano, catedrático de Nutrición Deportiva y especialista en Endocrinología y Nutrición, en el 5o Congreso Internacional de Frutos Rojos, organizado por Freshuelva y celebrado en junio de 2019. Apoyándose en el Índice ORAC (Oxygen Radical Absorbance Capacity), este especialista presenta las moras y los arándanos como:

\footnotetext{
"los dos alimentos con mayor capacidad antioxidante de la tierra", a lo que hay que añadir la destacada composición fitoquímica de los frutos rojos, lo que evidencia que "estamos vendiendo salud", puesto que son beneficiosos para "optimizar las funciones cerebrales y el sistema nervioso, prevenir el Alzheimer y el cáncer, controlar el estrés, retrasar el envejecimiento..." y un largo etcétera. (Freshuelva, 2019, párr. 4)
}

En un contexto en el que las preocupaciones por la salud son un motor importante en el crecimiento del consumo de productos frescos y en la conformación de la dieta postmoderna (Friedland, 1994), saberse dueños de una producción de estas características es valorado y sentido por el sector como una fortaleza: "Nosotros tenemos la fortaleza de trabajar con un producto que además de ser natural y no estar ultraprocesado, porque se puede comer en fresco o se puede comer en mermeladas que no son ultraprocesadas" (Begoña Mayoral, responsable de marketing de la cooperativa Onuberries) ${ }^{7}$.

En esta carrera por cultivar productos únicos destaca, junto al énfasis puesto en las ventajas nutricionales y saludables de los frutos rojos, la introducción de frutos considerados culturalmente exóticos. Aun gozando de menor grado de implantación, la agricultura onubense se suma progresivamente al cultivo de productos propios de zonas tropicales en los que se ha venido especializando la costa de Granda y Málaga. Junto al cultivo de frambuesas y arándanos, las explotaciones agrícolas del Grupo Medina se han especializado en la producción del ya mencionado caqui sharoni, el caqui Persimon y el kumquat. El caqui sharoni, variedad originaria históricamente de China y Japón, ha llegado a los campos de Huelva desde Israel. Presentado como "una deliciosa fruta exótica, dulce y sin semilla", es considerado su producto más emblemático, en la medida en que "representa fielmente nuestros valores de calidad e innovación" numerosas cooperativas y empresas de Huelva, de productos como la pitahaya o fruta del dragón y el physalis, también denominado alquejenje o farolillo chino, se incluye en esta práctica de diferenciación. En esta estrategia productiva el valor simbólico de lo exótico opera envolviendo el producto de los significados culturales ligados a un origen alejado geográfica y culturalmente, a la novedad y la escasez. Un valor simbólico que, del fruto, pasa a ser proyectado sobre el sector productivo e interpretado como una muestra más de su capacidad de sofisticación.

Comprender esta estrategia de diferenciación de la oferta obliga a retener distintos aspectos. Entre ellos, el objetivo de extender la campaña a lo largo de todo el año, aunque se logra bajo una especialización intensiva alejada del policultivo rotativo, así como el de reducir la dependencia de la producción y el precio de la fresa ante el auge de otros territorios productores. Igualmente, reorientar la producción hacia nichos de mercado distinguidos que, aún controlados por la gran distribución, permiten obtener mejores

\footnotetext{
7 Como observa Friedland (1994), la denominación de fresco como opuesto a transformado es una definición social que en la práctica hace referencia solo a su carácter perecedero, dado que cualquier alimento implica una transformación, una intervención de la acción humana. Esta apreciación es especialmente pertinente en un sector, como el de la agricultura industrial, en el que la innovación tecnológica juega un papel decisivo en todo el proceso, empezando por la investigación varietal que incluye la manipulación de semillas a partir de la biotecnología.
}

8 Web oficial del Grupo Medina. 
precios, constituye una forma de responder a ese aumento de la competencia. Si bien el recurso a la especialización y la innovación como base de la competitividad no son nuevas en la historia de este cultivo, hemos visto como en el último periodo han pasado a expresarse a través de nuevas prácticas y significados culturales que se articulan con el concepto de calidad.

"Las mercancías son buenas para pensar", afirma Narotzky (2004, p. 154), y no pueden ser abstraídas de las relaciones sociales que las producen, de ahí la importancia de considerar la apuesta activa y dinámica por productos únicos, saludables y exóticos en relación con los contextos económicos, sociales y culturales en los que se despliega. En la lucha por mejorar la posición del sistema productivo local en los mercados globales a partir de la especialización en este tipo de productos, el liderazgo es entendido en términos de volumen de exportación, renta monetaria y capital simbólico. En el mercado de bienes agroalimentarios, ajustado cada vez más a los gustos distinguidos (Bourdieu, 1979), la acumulación de marcas de distinción, basadas en la idea de singularidad, deviene una estrategia fundamental de acumulación de capital (Harvey, 1998, 2007). De esta forma, el sector productivo se suma a la tendencia global propia del modelo postfordista que sitúa en el centro la distinción y la innovación, y las vincula a la calidad a partir de la imagen de marca del producto (Roseberry, 1996; Alonso, 2007).

Estas estrategias y expectativas, sin embargo, no está exentan de contradicciones y limitaciones. Como han constatado estudios empíricos realizados en otros territorios orientados a la diferenciación de la producción en fresco, si bien se introducen rupturas significativas de la mano del postfordismo, la producción de escala sigue siendo un pilar fundamental del modelo, la diferenciación obtenida en la práctica es elemental y la supeditación a parámetros estandarizados impuestos por los clientes aumenta (Lara, 1999; Pedreño, 2001; Friedland, 1994). Las creencias y expectativas depositadas en la innovación y la distinción volverán a ocupar un lugar estratégico en otra de las estrategias desarrolladas por el sector para alcanzar competitividad, el ensayo de variedades de calidad desde el ámbito local.

\section{'Variedades locales para un mercado global': la investigación varietal como instrumento de calidad}

Luis García, un productor de 46 años originario de Puerto Serrano, un pueblo de la Sierra de Cádiz con una fuerte tradición emigratoria, rememora durante la entrevista su pasado jornalero. Llegó a los campos de fresas en el marco de las migraciones temporales que cada año emprendían las familias jornaleras andaluzas para cubrir distintas campañas agrícolas. Trabajó a jornal en el cultivo de la fresa hasta que conoció a su actual esposa y pasó a trabajar con ella en la finca que tenían sus suegros. Estos comenzaron cultivando fresas en una explotación de una hectárea y media, extensión que fue aumentando en tamaño hasta alcanzar las 24 hectáreas que ha pasado a gestionar Luis tras la jubilación de sus suegros. Sentados en torno a la mesa de una oficina situada en el almacén del campo, Luis y sus dos hijos, que también trabajan en la empresa familiar, hablan sobre el papel que ha tenido la tecnología en la evolución del cultivo. "Como decía mi suegro antes, ¿no?, con el mulo se ponía el plástico, se acolchaba el suelo, ¿no? Y hoy en día pues hay tecnología para todo". Entre los avances tecnológicos incluye los radiadores avanzados, los ordenadores, el riego automático y, más recientemente, la tecnología para controlar la calidad de la producción. Una de las últimas innovaciones tecnológicas en la que han invertido es, precisamente, la línea de manipulado y selección de la fruta instalada en el almacén del campo para garantizar que la fruta que sale a la cooperativa sea de máxima calidad. Al explicar en qué se basa la calidad, Luis apunta el papel que la biotecnología desempeña en este terreno: 
La calidad, pues, aparte de, del tema saludable, ¿no?, de, de los químicos o lo que podíamos decir. Pues también de una fruta que creo que, que sea capaz de llegar al mercado en condiciones, que tenga un buen sabor, una buena vista. Todo eso influye, claro. Y por eso se están buscando variedades que, que intenten llegar de esa manera.

Entre esas variedades que "se están buscando" sobresalen Rociera y Rábida, investigadas por la empresa onubense Fresas Nuevos Materiales. En la campaña 2019/20 Luis ha optado por combinar en su explotación las variedades de fresas procedentes de la Universidad de Florida, como Fortuna y Brilliance, con aquellas otras creadas en Huelva. En esa orientación hacia la investigación varietal local se encuentra también la apuesta que Luis ha hecho por Cupla, una variedad temprana de arándanos patentada por la cooperativa de la que es socio. Una variedad que "es nuestra", que "es propia".

La investigación en biogenética es interpretada, desde los inicios del modelo, como signo de la modernización del cultivo respecto de la agricultura tradicional practicada en el litoral onubense hasta la llegada de la agricultura industrial. El incremento de la productividad de la planta, la capacidad de resistencia de la fruta y la producción extra-temprana son algunas de las transformaciones introducidas por la investigación en biogenética celebradas por el sector. La creación, en las últimas dos décadas, de departamentos dedicados a I + D en las cooperativas y grupos empresariales y en los organismos públicos, así como la formación de empresas privadas vinculadas al sector y orientadas en esta dirección, muestran el impulso dado desde el contexto local a la investigación varietal. Los últimos diez años revelan dos cambios de hondo calado: se consolidan las iniciativas y esfuerzos por reducir la dependencia de las entidades extranjeras en el terreno de la investigación en biotecnología y se pasa a asociar progresivamente esta última con la calidad.

Fresas Nuevos Materiales (FNM), creada en Huelva en 1999 (Silva, 2011) y en la que el sector productivo -a través de Fresas Investigación y Desarrollo S.A-tiene una participación del $50 \%$, constituye uno de los ejemplos más representativos de los avances logrados en este sentido. Coral, la primera variedad propia registrada en 2005, en un periodo en el que el mercado de variedades estaba dominado por Estados Unidos, ha dado paso a tres nuevas variedades: Primoris, Rábida y Rociera9. Situarse, en la campaña 2017/2018, en la segunda posición (33,9\%) en variedades empleadas en Huelva, sólo superada por el 37\% de la Universidad de Florida ${ }^{10}$, es percibido como una muestra clara del carácter emprendedor y profesional del sector, entendido éste bajo la lógica de competir y ganar terreno a otras empresas y regiones:

\footnotetext{
[Se trata de] una política varietal totalmente distinta. El material vegetal español también, bueno, ha sido... En el Congreso ayer se comentaba, en la última jornada, ha sido muy importante esa dependencia que teníamos del material americano. (...) Hace quince años pusimos un capital para investigar, para crear una empresa [FNM] que investigara variedades hechas por y para Huelva. Hechas en Huelva. Y de ahí ha salido, después de quince años, ahora tenemos dos variedades [de fresas], una principalmente, Rociera, y otra que se llama Rábida, que son las que se están comiendo prácticamente todo el material vegetal que viene de Florida, que viene de California, de Francia, de donde quiera que venga. (...) Te puedo decir que un año normal nos podemos ahorrar en royalties ahora mismo doscientos o trescientos mil euros (Domingo Gutiérrez, gerente de la cooperativa Onuberries).
}

Si con el ensayo de variedades se persigue reducir la dependencia externa, el coste económico que entraña el pago de royalties y crear variedades únicas que se ajusten plenamente a las condiciones del cultivo en Huelva (tipo de suelos, clima), también se busca complementar la productividad con la mejora de la calidad. Ésta es definida en relación con la conservación, la presencia formal, el aroma y el sabor. Los criterios que maneja Fresas Nuevos Materiales para seleccionar las variedades que finalmente se

9 En 2013 iniciaron un programa de investigaciones de variedades de frambuesas.

10 Datos extraídos de la web de la empresa Fresas Nuevos Materiales. 
comercializan así lo reflejan: precocidad, tolerancia a enfermedades, firmeza, color interno y externo, homogeneidad del fruto, forma y producción ${ }^{11}$. Lucía Ruiz, licenciada en biología y empleada desde hace dos años como técnica de calidad en la empresa Fresas Moreno, subraya la relevancia de la investigación varietal y la sitúa en relación con esta concepción de la calidad:

\begin{abstract}
Lo que nos diferencia del resto es que nosotros damos calidad, que te aseguramos que la fruta mm... la quieres así, nosotros te la vamos a dar así. Además, tenemos una marca propia. Una variedad propia, Candela. (...) Tenemos un departamento de I+D que se encarga de desarrollar nuevas variedades y ya tenemos patentado una de ellas y comercializamos con ella, la verdad. Y la, es la que mejor está de todas las que tenemos, la verdad. (...) Que es una fresa que tú te la... es dulce, es crujiente, después, es súper uniforme, la tonalidad es... Te voy a enseñar unas fotos.
\end{abstract}

Estos testimonios expresan las lógicas que orientan las prácticas económicas que impulsa el sector y el marco cultural en el que se inscriben. Un marco en el que la biotecnología aparece ligada, además de a la productividad, a la calidad, la innovación, el control y la seguridad (Martínez, 2019). El propio concepto de semilla mejorada revela el modo en que la investigación en biogenética es percibida como una mejora (en presencia, conservación, producción y sabor) respecto a la calidad de las variedades tradicionales. La idea de calidad se halla integrada, simultáneamente, por valores que apelan a la producción natural, saludable y en fresco (no procesada) y por valores que ensalzan la producción industrial y biotecnológica, actualizando así el imaginario de la modernización apoyado en los avances de la tecnología y la ciencia y asociado a la idea de progreso (Delgado, 2002; Naredo, 2006). En este sentido, debemos tener presente que la creciente organización del sistema alrededor de un conjunto de preocupaciones basadas en la conveniencia, la salud y el bienestar, en la producción en fresco y en la innovación no puede llevarnos a pensar lo natural frente a lo industrial (ni lo orgánico frente a lo convencional), pues no se trata de modelos ideales en oposición, sino de elementos que forman parte de un único régimen alimentario (Burch y Lawrence, 2009). Un régimen en el que la biotecnología aparece vinculada, además, a la capacidad de control (sobre la naturaleza, el clima y los ecosistemas) y a la garantía de seguridad (respecto a posibles enfermedades y plagas) ${ }^{12}$. La creciente relevancia que adquiere la seguridad alimentaria bajo el postfordismo ayuda a entender cómo su vinculación con la calidad influye en el desarrollo de la biotecnología (Sorj y Wilkinson, 1994), en la medida en que esta es considerada un elemento determinante de la obtención de calidad (Martínez, 2014).

El objetivo de crear "variedades locales para un mercado global" no significa, en este contexto, recuperar semillas autóctonas seleccionadas año tras año mediante métodos tradicionales, vinculadas históricamente a un territorio y, con ello, a ecosistemas, técnicas de cultivo, dietas alimentarias y culturas locales. El marco social en el que adquiere sentido este eslogan de la empresa Fresas Nuevos Materiales es bien distinto. Las variedades locales son aquellas que se distinguen por ser "hechas por y para Huelva", como afirmaba el gerente de la cooperativa Onuberries, pero en laboratorios, siguiendo los procedimientos y criterios estandarizados que emplean otras empresas y territorios, variedades dependientes de insumos externos, fertilizantes, pesticidas y grandes cantidades de agua de riego, y sujetas, tras ser patentadas, al pago de royalties.

Esta concepción que se tiene de las variedades locales y la calidad se aleja radicalmente de otras propuestas de recuperar las variedades y semillas locales, como las realizadas en el marco del paradigma de la soberanía alimentaria. Organizaciones sociales e

11 Web de la empresa Fresas Nuevos Materiales.

12 Véase Stuart (2008) para un análisis sobre la relación entre agricultura intensiva y multiplicación de enfermedades, plagas y riesgos para la salud y el medio ambiente, y sobre cómo a través de la innovación tecnológica la industria más bien crea una ilusión de control. 
investigaciones académicas definen las variedades locales y su relación con la calidad a partir de otro tipo de parámetros y significados culturales: el carácter autóctono de las semillas, el empleo de métodos tradicionales en el proceso de selección y recuperación, su vinculación a ecosistemas y cultivos locales, su adaptación al sistema de policultivo, el respeto de la biodiversidad, el uso de canales cortos y prácticas agroecológicas (Acosta, 2007; Soler Montiel y Calle, 2010; Rosset, 2018; Luetchford, 2019). Otro elemento importante en esta concepción es la interpretación de las variedades autóctonas como un modo de construir, desde el campesinado y la agricultura familiar, una autonomía relativa frente a las grandes empresas que investigan y distribuyen variedades comerciales. Al definirlas como comerciales, el paradigma de la soberanía alimentaria pone énfasis en el peso que en ellas tiene el valor monetario y el objetivo del lucro, en detrimento de los objetivos sociales.

Debe retenerse que al apostar por la investigación varietal desde el ámbito local el sector productivo de Huelva busca alcanzar cierta autonomía frente a las grandes empresas que hasta ahora han dominado el mercado, pero busca alcanzarla desde el paradigma de la globalización agroalimentaria. En su trabajo de asesoría y colaboración con la Junta de Andalucía, Castells y Hall (1994) se referían a las limitaciones de una economía regional basada en la especialización de la agricultura de exportación en un sistema en el que las plusvalías se concentran en quienes detentan la capacidad de innovación tecnológica. Como ejemplo señalan la tendencia a que los beneficios esenciales sean para las compañías capaces de generar nuevas semillas y no para los agricultores que las utilizan. Desde un tono optimista próximo al determinismo tecnológico y ajeno a otros factores históricos fundamentales, un tono del que también participan los autores citados, el sector productivo aspira a terminar con esas relaciones de subordinación a partir del dominio de la investigación aplicada y la innovación tecnológica.

\section{CONCLUSIONES}

El análisis realizado ha mostrado cómo piensan y actúan distintos actores del sector productivo de frutos rojos en Huelva en relación con la calidad, ilustrando algunos de los ajustes que se están llevando a cabo para adaptarse a la fase actual de globalización agroalimentaria. La aproximación empírica ha revelado la manera en que el discurso y las prácticas en torno a la calidad no sólo están presentes en las certificaciones estandarizadas destinadas específicamente a gestionarla, sino que permean un conjunto de estrategias más amplias. En este sentido, la mirada histórica y localizada en el territorio ha permitido comprender los cambios que experimenta y el modo en que se actualiza, bajo el objetivo de la calidad, el nexo entre diferenciación del producto y especialización productiva e investigación varietal.

En primer lugar, sobresale la reorientación que experimenta la especialización productiva hacia la diferenciación de la oferta, y cómo en esta estrategia la unicidad, la novedad y la salud se articulan con el concepto de calidad. En segundo lugar, e íntimamente relacionado, destaca la relevancia concedida al dominio y aplicación de la biotecnología desde el ámbito local para mejorar la calidad de la fruta, entendida ésta en relación con la presencia (uniformidad, calibre, color), conservación, aroma y sabor. El significado particular que adquiere, en el marco de estas estrategias, el concepto de variedad local manejado por el sector o la idea de unicidad movilizada bajo la estrategia de especialización refleja cómo la actividad económica es regulada por valores sociales y culturales que se articulan con el valor monetario. Esto es, cómo la calidad no se configura en abstracto, sino en un modelo de agricultura industrial que, aún apoyada en la producción de escala, integra 
estrategias de diferenciación propias del postfordismo, en las que la calidad se convierte en un parámetro de competitividad y se construye en relación con las ideas de innovación y distinción.

El análisis ha evidenciado, igualmente, que los actores locales participan activamente en la construcción del sistema de calidad, aun en el marco de estructuras y tendencias globales atravesadas por relaciones de poder. Si la búsqueda de innovación y distinción ha introducido ciertos cambios en las estrategias productivas, esto ha ido acompañado de un aumento de la supeditación del territorio a las nuevas exigencias y agentes externos.

\section{AGRADECIMIENTOS}

El artículo presenta los resultados del proyecto "Gobernanza de la calidad en las cadenas globales agroalimentarias. Un análisis comparado de los territorios agro-exportadores en España", financiado por el Ministerio de Ciencia e Innovación y Fondos FEDER de la Unión Europea (CSO2017-85507-P).

\section{REFERENCIAS}

Acosta, R. (2007). La biodiversidad en la agricultura. La importancia de las variedades locales. En J. Maestre, A. González y Á. Casas (eds.), Nuevas rutas para el desarrollo en América Latina. Experiencias globales y locales (pp. 234-255). México D. F.: Universidad Iberoamericana.

Alonso, L. E. (2007). Las nuevas culturas del consumo y la sociedad fragmentada. Pensar la Publicidad, 1(2), 13-32.

Bergeron. E. y Darpeix, A. (2004). Desarrollo y límites del sistema fresero intensivo en mano de obra del Condado del Litoral, Provincia de Huelva. París: Institut National Agronomique.

Bonanno, A. y Cavalcanti, J.S. (2012). Globalization, Food Quality and Labor: The Case of Grape Production in North-Eastern Brazil. International Journal of Sociology of Agriculture and Food, 19(1), 37-55.

Bourdieu, P. (1979). La distinción. Criterios y bases sociales del gusto. Madrid: Taurus.

Burch, D. y Lawrence, G. (2009). Towards a third food regime: Behind the transformation. Agriculture and Human Values, (26), 267-279. https://doi.org/10.1007/s10460-009-9219-4

Castells, M. y Hall, P. (1994). Tecnópolis del mundo. La formación de los complejos industriales del siglo XXI. Madrid: Alianza.

Delgado, M. (2002). Andalucía en la otra cara de la globalización. Sevilla: Mergablum.

Delgado, M. y Aragón, M. A. (2006). Los campos andaluces en la globalización. Almería y Huelva, fábrica de hortalizas. En M. Etxezarreta (ed.), La agricultura española en la era de la globalización (pp. 423-474). Madrid: Ministerio de Agricultura y Pesca.

Farruggia, D., Crescimannoa, M., Galatia, A. y Tinervia, S. (2016). The quality perception of fresh berries: An empirical survey in the German market. Agriculture and Agricultural Science Procedia, 8, 566-575. https://doi.org/10.1016/j.aaspro.2016.02.075 
Fernández, I. (2018) Más de 60 años a la vanguardia del sector. Grupo Medina. Revista Mercados (13/04/2018). https://revistamercados.com/articulo/mas-de-60-anos-a-lavanguardia-del-sector-grupo-medina/

Freshuelva. (2017). El boom de los frutos rojos. Distribución y Consumo, 3, 33-35.

Freshuelva. (2019). El 5o Congreso Internacional de Frutos Rojos pone el acento en las propiedades nutricionales y el enorme potencial antioxidante de los frutos rojos como nuevos valores de venta. Recuperado de https://freshuelva.es/el-5o-congresointernacional-de-frutos-rojos-pone-el-acento-en-las-propiedades-nutricionales-y-elenorme-potencial-antioxidante-de-los-frutos-rojos-como-nuevos-valores-de-venta/

Friedland, W. (1994). La nueva globalización: el caso de los productos frescos. En A. Bonanno (ed.), La globalización del sector alimentario (pp. 275-321). Madrid: MAPA.

Friedland, W. (2004). Agrifood globalization and commodity systems. International Journal of Sociology of Agriculture and Food, 12, 1-12.

Friedmann, H. y McMichael, P. (1989). Agriculture and the state system: The rise and decline of national agricultures, 1870 to the present. Sociologia Ruralis, 29(2), 93-117.

Goodman, D. y Watts, M. (1997). Agrarian questions. Global appetite, local metabolism: Nature, culture and industry in fin-de-siècle agro-food systems. En D. Goodman y M. J. Watts (eds.), Globalising food. Agrarian questions and global restructuring (pp. 1-34). Londres/Nueva York: Routledge.

Harvey, D. (1998). La condición de la posmodernidad. Buenos Aires: Amorrortu.

Harvey, D. (2007). Espacios de esperanza. Madrid: Akal.

Junta de Andalucía. (2019). Observatorio de Precios. Sevilla: Junta de Andalucía.

Konefal, J., Mascarenhas, M. y Hatanaka, M. (2005). Governance in the global agro-food system: Backlighting the role of transnational supermarket chains. Agriculture and Human Values, 22, 291-302. https://doi.org/10.1007/s10460-005-6046-0

Lacomba, J. A. (1997). La agricultura mediterránea andaluza y California, de finales del XIX a principios del XX: permanencias y cambios en una fase de transformaciones. En J. Morilla, J. Gómez-Pantoja Fernández-Salguero y P. Cressier (eds.), Impactos exteriores sobre el mundo rural mediterráneo: del Imperio Romano a nuestros días (pp. 473-494). Madrid: Ministerio de Agricultura, Alimentación y Medio Ambiente.

Lara, S. (1998). Nuevas experiencias productivas y nuevas formas de organización flexible del trabajo en la agricultura mexicana. México: Juan Pablo Editores.

Lawrence G. y Dixon J. (2016). The political economy of agri-food: Supermarkets. En A. Bonanno y G. Lawrence (eds.), Handbook of the International Political Economy of Agriculture and Food (pp. 213-231). Cheltenham/Northampton: Edwar Elgar Publishers.

Lozano, A. (2019) Los frutos rojos de Huelva ya no son solo volumen, sino calidad. Revista Mercados (11/03/2019). https://revistamercados.com/articulo/los-frutos-rojos-dehuelva-ya-no-son-solo-volumen-sino-calidad-2/]

Luetchford, P. (2019). What's in a Just Price? Challenging Values at an Organic Cooperative in Southern Spain. Research in Economic Anthropology, 39, 91-112. https://dx.doi.org/10.1108/S0190-128120190000039005

Marsden, T. (1997). Creating space for food: The distinctiveness of recent agrarian development. En D. Goodman y M. J. Watts (eds.), Globalising food. Agrarian questions and global restructuring (pp. 169-191). Londres/Nueva York: Routledge. 
Marsden, T. (2012). Third Natures? Reconstituting Space through Placemaking Strategies for Sustainability. International Journal of Sociology of Agriculture and Food, 19(2), 257-274.

Marsden, T. y Arce, A. (1995). Constructing quality: Emerging food networks in the rural transition. Environment and Planning A, 27, 1261-1279.

Martínez, B. (junio, 2014). About moral economy: Fair price, quality and sustainability in Galician farming exploitations. Trabajo presentado en Value and values in agro-food processes, Marsella, Francia.

Martínez, B. (2019). The Moral Price of Milk: Food Values and the Intersection of Moralities and Economies in Dairy Family Farms in Galicia. In V. Siniscalchi y K. Harper (eds.), Food values in Europe. London/New York: Bloomsbury Academic.

McMichael, P. (1996). Globalization: Myths and Realities. Rural Sociology, 61(1), 25-55. https://doi.org/10.1111/j.1549-0831.1996.tb00609.x

McMichael, P. (2011). Food system sustainability: Questions of environmental governance in the new world (dis)order. Global Environmental Change, 21(3), 804-812. https://doi.org/10.1016/j.gloenvcha.2011.03.016

Moraes, N. y Cutillas, I. (2014). Nuevos dispositivos de regulación transnacional: un análisis sobre los estándares de calidad y responsabilidad social y su impacto en los enclaves globales agrícolas. En A. Pedreño (coord.), De cadenas, migrantes y jornaleros. Los territorios rurales en las cadenas globales agroalimentarias (pp.120-135). Madrid: Talasa Ediciones.

Moreno, J. (2009). Los contratos en origen de temporada: mujeres marroquíes en la agricultura onubense. Revista de Estudios Internacionales Mediterráneos, 7, 58-78.

Moreno, J. (2016). Trabajo y género en la globalización agroalimentaria: las trabajadoras de la fresa en Marruecos. Tesis doctoral inédita, Departamento de Anropología Social, Universidad Autónoma de Madrid.

Naredo, J. M. (2006). Raíces económicas del deterioro ecológico y social. Más allá de los dogmas. Madrid: Siglo XXI.

Narotzky, S. (2004). Antropología económica. Nuevas tendencias. Barcelona: Melusina.

Pedreño, A. (1999). Del jornalero agrícola al obrero de las factorías vegetales. Madrid: Ministerio de Agricultura, Pesca y Alimentación.

Pedreño, A. (2001). Efectos territoriales de la globalización. El caso de la ruralidad agroindustrial murciana. Revista de Estudios Regionales, 59, 69-96.

Ponte, S.yGibbon, P.(2005). Quality standards, conventions and the governance of global value chains. Economy and society, 34(1), 1-31. https://doi.org/10.1080/0308514042000329315

Pitarch, J. (2015) La fresa española debe avanzar en comunicación para ganar competitividad (Entrevista a Carlos Cumbreras, gerente de Grufesa). FreshPlaza (27/01/2015). https://www.freshplaza.es/article/3087364/la-fresa-espanola-debe-avanzar-encomunicacion-para-ganar-competitividad

Ransom, E., Bain, C. y Higgins, V. (2013). Private Agri-food Standards: Supply Chains and the Governance of Standards. International Journal of Sociology of Agriculture and Food, 20(2), 147-154.

Redacción (2016) Cuna de Platero comienza una nueva campaña de frutos rojos. Origen. La revista del sabor rural (08/09/2016). https://www.origenonline.es/index. php/2016/09/08/cuna-platero-comienza-una-nueva-campana-frutos-rojos/ 
Reigada, A. (2012). Más allá del discurso sobre la 'inmigración ordenada': contratación en origen y feminización del trabajo en el cultivo de la fresa en Andalucía. Política y Sociedad, 49(1),103-122.

Reigada, A. (2016). Family farms, migrant labourers and regional imbalance in global agrifood systems. On the social (un)sustainability of intensive strawberry production in Huelva (Spain). En A. Corrado, D. Perrota, C. de Castro (Eds.), Migration and Agriculture: Mobility and Change in the Mediterranean Area (pp.95-110). London: Routledge.

Renard, M. C. (2003). Fair Trade: Quality, Market and Conventions. Journal of Peasant Studies, 19, 87-96. http://dx.doi.org/10.1016/S0743-0167(02)00051-7

Roseberry, W. (1996). The Rise of Yuppie Coffees and the Reimagination of Class in the United States. American Anthropologist, 98(4), 762-775. https://doi.org/10.1525/ aa.1996.98.4.02a00070

Rosset, P. (2018). Las semillas representan una soberanía sobre el proceso productivo. Entrevista a Peter Michael Rosset. Revista Ae. Semillas y Agroecología, 33, s. p.

Salas, J. y Flores, A. (1985). El cultivo del fresón en la costa de Huelva. Sevilla: Consejería de Agricutlura y Pesca.

Silva, R. (2011). Territorio, redes e innovación en el sistema agrocomercial de la fresa de Huelva. Cuadernos de Estudios Agroalimentarios, 2, 109-131.

Soler Montiel, M. y Calle, A. (2010). Rearticulando desde la alimentación. Canales cortos de comercialización en Andalucía. En M. Soler Montiel, C. Guerrero (coords.) y R. FernándezVaca (dir.), Patrimonio Cultural en la Ruralidad Andaluza (pp. 258-283). Sevilla: Junta de Andalucía.

Sorj, B. y Wilkinson, J. (1994). Biotecnologías, multinacionales y sistemas agroalimentarios de los países en desarrollo. En A. Bonanno (ed.), La globalización del sector alimentario (pp. 175-199). Madrid: MAPA.

Stuart, D. (2008). The illusion of control: Industrialized agriculture, nature, and food safety. Agriculture and Human Values, 25, 177-181. https://doi.org/10.1007/s10460-008-9130-4

Veltz, P. (1999). Mundialización, Ciudades y Territorios. Barcelona: Ariel.

\section{NOTA BIOGRÁFICA}

Alicia Reigada es Profesora Contratada Doctora en el Departamento de Antropología Social de la Universidad de Sevilla y miembro del Grupo de Investigación GEISA. Sus estudios se han centrado en el análisis de las cadenas globales agrícolas, la organización social del trabajo y las migraciones desde una perspectiva feminista. Ha participado en diferentes proyectos de investigación y redes internacionales, y ha realizado estancias de investigación en la City University of New York (CUNY), University of California, Davis, Universidad Nacional Autónoma de México (UNAM), Universidad Federal Rural de Río de Janeiro (UFRRJ), Flacso-Ecuador, entre otras.

Juana Moreno Nieto es profesora sustituta interina en la Universidad de Cádiz. Licenciada en Sociología y Doctora en Antropología Social por la Universidad Autónoma de Madrid. Ha dedicado la mayor parte de su carrera académica al estudio de las transformaciones en el sistema agroalimentario globalizado, la organización del trabajo y las migraciones en áreas 
de agricultura intensiva desde una perspectiva feminista. Ha realizado investigaciones sobre estas cuestiones en Marruecos, España y Francia. Ha realizado diversas estancias en universidades y centros extranjeros y entre 2016 y 2018 ha sido investigadora postdoctoral en el LEST y el Centro Norbert Elias (Universidad Aix Marsella-CNRS-EHESS, Francia).

Carmen Mozo es Profesora Titular en el Departamento de Antropología Social de la Universidad de Sevilla y miembro del Grupo de Investigación GEISA. Su trayectoria investigadora ha articulado el interés por la implementación de las perspectivas feministas, inicialmente en el ámbito laboral, con la Antropología de la Salud, campo en el cual ha desarrollado sus últimas investigaciones. 